\title{
Neo-SENSE: a non-invasive smart sensing mattress for cardiac monitoring of babies
}

\author{
$1^{\text {st }}$ Rodrigo Aviles-Espinosa \\ School of Engineering and informatics \\ University of Sussex \\ Brighton, United Kingdom \\ R.Aviles-Espinosa@sussex.ac.uk \\ $4^{\text {th }}$ Henry Dore \\ School of Engineering and informatics \\ University of Sussex \\ Brighton, United Kingdom \\ hd255@sussex.ac.uk \\ $7^{\text {th }}$ Robert J.Prance \\ School of Engineering and informatics \\ University of Sussex \\ Brighton, United Kingdom \\ r.j.prance@sussex.ac.uk
}

\author{
$2^{\text {nd }}$ Elizabeth Rendon-Morales \\ School of Engineering and informatics \\ University of Sussex \\ Brighton, United Kingdom \\ er241@sussex.ac.uk \\ $5^{\text {th }}$ Oana Anton \\ Academic Department of Paediatrics, \\ Royal Alexandra Children's Hospital \\ Brighton, United Kingdom \\ Oana.anton@bsuh.nhs.uk
}

\author{
$3^{\text {rd }}$ Zhenhua Luo \\ School of water, Energy and \\ Environment \\ Cranfield University \\ Cranfield, United Kingdom \\ Z.Luo@cranfield.ac.uk \\ $6^{\text {th }}$ Heike Rabe \\ Academic Department of Paediatrics, \\ Royal Alexandra Children's Hospital \\ Brighton, United Kingdom \\ Heike.Rabe@bsuh.nhs.uk
}

\begin{abstract}
Within the first minute of life a newborn must take its first breath to make the transition from life inside the womb to the outside world. If a baby does not start breathing, its heart rate will drop and the circulation of blood carrying oxygen to the organs will be seriously affected. The damage done to a newborn who is deprived of oxygen happens so quickly that rapid response is imperative. During birth, the attending neonatal staff manually listen to the baby's heart and count the heart rate; however, this has proven inaccurate and inefficient. Nowadays, there is not a reliable method to monitor newborn heart rate promptly throughout birth. In this paper, we report the design and development of a novel smart mattress device to measure the babies' electrocardiogram and respiration non-invasively. The device is based on electrometer-based amplifier sensors combined with novel screen-printing techniques. Proof of concept tests are carried out to demonstrate the suitability of the smart-mattress for new born ECG monitoring. We perform tests with a young infant and demonstrate the potential of this sensing technology to provide a quick and reliable application as ECG readings were displayed within a time $<30$ seconds. This will aid the neonatal staff to assess the success of the resuscitation technology aiming to lower the incidence of long-term consequences of poor adaptation to life outside the womb.
\end{abstract}

Keywords-Biosensors, medical sensors, pediatric cardiology, Electric Potential Sensors, electrophysiology and electrocardiogram.

\section{INTRODUCTION}

Within the first minute of life, a newborn must take its first breath to make the transition from life inside the womb to the outside world. Each year, there are approximately 135 million births globally (UN, 2011) and 0.8 million births in the UK. Approximately $10 \%$ of these babies require assistance during this transition. If a baby does not start breathing, its heart rate will drop and the circulation of blood carrying oxygen to the organs will be seriously affected. Neonatal staff are requird to stimulate these babies to breathe immediately though resuscitation procedures. Failure to do so will result in newborn brain injuries such as cerebral palsy and impaired motor skills which could harm the baby's longterm lifetime.

In a study carried out to assess the response to resuscitation of the new born [1], it has been reported that out of 591 births all of which required resuscitation, $30 \%$ of the infants experienced some level of brain injury due to oxygen deprivation, of which $30 \%$ died in the first week following birth. According to the World Health Organization, globally there are 13.5 million of resuscitations, 4 million affected by brain injuries and nearly 2.5 million deaths [2]. However, due to the lack of facilities and staff in many locations, especially in developing countries these numbers are likely to be much higher.

Current qualitative methodologies to evaluate the resuscitation procedure efficacy such as palpation and auscultation rely on the experience of the neonatal staff attending the birth. Within the last two decades, technologies that allow measuring the heart rate (HR) of the newborns have been introduced. These technologies include: pulse oximetry (PO), electrocardiography, Doppler ultrasound and forehead reflectance photo plethysmography (PPG). Among all these, ECG and PO have shown to be more precise than qualitative methodologies. However, multiple studies have shown that using PO $[3,4]$, the time elapsed after birth to the successful establishment of HR display often exceeds one to two minutes.

Moreover, a limitation of PO is that HRs $<100 \mathrm{bpm}$ in newborns especially preterm infants are not consistently detected due to the weakness of blood perfusion [5].

The current challenges of preterm infant monitoring combined with increasingly sophisticated scientific advancements, have driven the development of novel technologies to assess both new-born and infant HR in ways that seek to overcome the limitations of currently available devices [6]. 


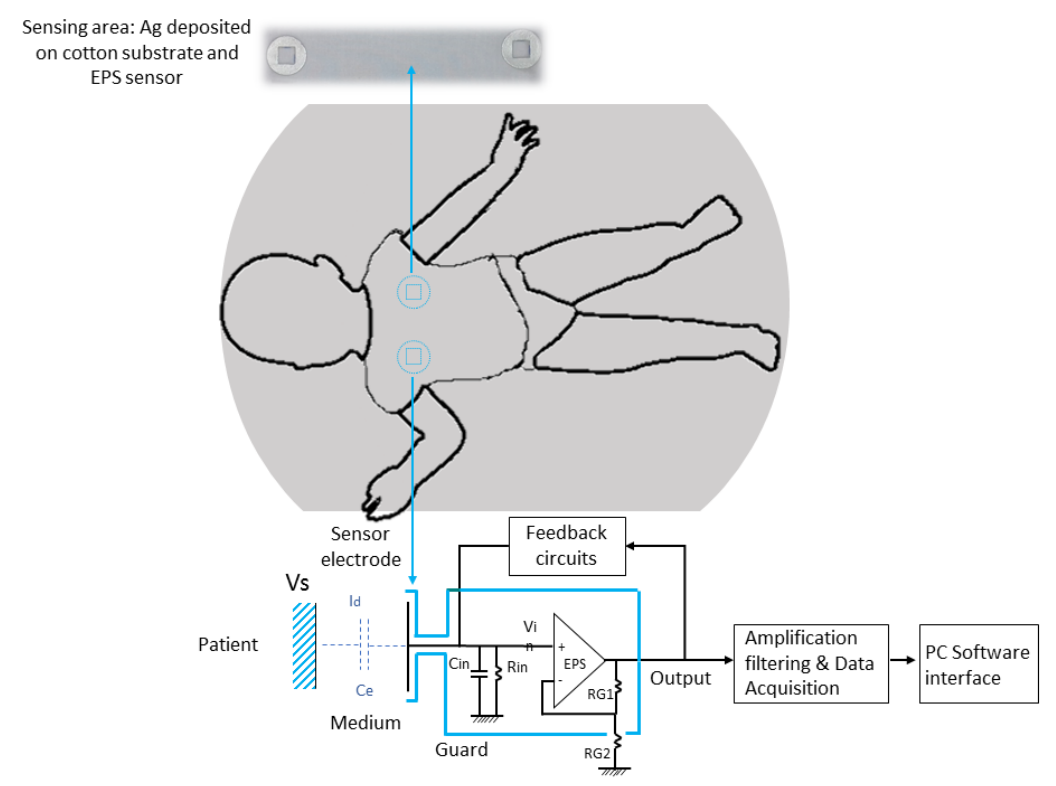

Fig. 1. Schematic of the smart-matress (top) and sensor design implementing the EPS sensor (bottom).

This is also supported by Finn et al. [5] where it is concluded that in order to provide an enhanced monitoring of preterm infants during stabilization in the delivery room, the electrocardiogram (ECG) monitoring should be available as an adjunct to ongoing clinical assessment.

Based on this fact, a fast and reliable method to monitor HR immediately after birth does not currently exist. Today's technologies lack both, accuracy and rapid application, and have not been designed considering the human factors associated with either term or preterm babies. This includes the newborn skin vulnerability, size and weight, which could be less than $1 \mathrm{~kg}$ in preterm infants.

Based on this clinical need, in this paper, we propose a novel health care solution based on an innovative smart sensing device to assist midwives and neonatal staff during baby delivery. We aim to tackle the challenge of accurately measuring the new-born's HR within the first minute of life and beyond. This is carried out by placing the newborn baby on top of a sensor-integratedsmart mattress, that provides ECG readings within a few seconds using non-invasive Electric Potential Sensors (EPS).

\section{ELECTRIC POTENTIAL SENSOR}

The EPS was invented at the University of Sussex as a non-invasive sensing technology [7]. Such sensor is a feedback enhanced and stabilized electrometer-based amplifier that operates based on displacement current measurements. Technical details on the sensor characterization have been extensively described elsewhere [8-12].

The smart sensing device was built in a neonatal mattress employing bespoke EPS sensors that included associated feedback loops providing the functions of guarding, bootstrapping, and neutralization to enhance the input impedance, reduce the input capacitance, and maintain the electronic stability of the sensor [8]. The net effect of this combined with positive feedback techniques is to produce a broadband sensor (up to $100 \mathrm{MHz}$ ), with extremely high input impedance (up to $10^{18} \Omega$ ) and low effective input capacitance $\left(\sim 10^{-15} \mathrm{~F}\right)$ which is crucial for weak capacitive coupling with the baby. This new device produces a very low noise floor sensor at the operating frequencies of electrophysiological signals such as fetal and neonatal ECG [13]. Its performance as a non-perturbative detector for measuring fields or voltages with high sensitivity level has proven a maximum sensitivity of $\sim 2.6 \mu \mathrm{V} / \mathrm{m}$ and an associated accuracy of $2 \%[11,12]$.

In our previous work, the EPS technology has been successfully tested for monitoring fetal ECG during early pregnancy (starting from week twenty of gestational age) [9] as well as recording ECG signals from micron sized living organisms such as Zebrafish [10]. This confirms that the sensor sensitivity and signal to noise ratio (SNR) is suitable to be used with preterm babies aged as early as 25 weeks having greater ECG amplitude given that the electrical signal does not need to propagate though maternal tissue.

\section{A. Experimental Setup}

The prototype described in this paper was built using an ultra-high input impedance EPS sensor with internal input bias current circuitry and guarding. The prototype was designed using a pair of dry EPS electrodes. The circuit design incorporates an electrometer and utilizes the electronic feedback techniques in order to increase the input impedance and maintain sensor stability. Figure 1 shows the schematic of the EPS sensor design along with the experimental setup designed to test the smart-mattress prototype for neonatal ECG monitoring.

We performed modifications on a mattress used in the neonatal intensive care unit (NICU) to include a pair of bespoke electro active EPS sensors. The voltage outputs from the sensors were sent to an analogue processing stage as shown in Figure 1, where signals were amplified, and conditioned by noise reduction (i.e. rejecting external noise at particular frequencies $<50 \mathrm{~Hz}$ ) and bandpass filtering. In this particular design, filters can be switched on/off for the detection of additional parameters such as breathing rates. The analogue output is digitized using a commercial 
National Instruments data acquisition system NI USB 6003 having a maximum sampling rate of $100 \mathrm{kS} / \mathrm{s}$. The data is then acquired using a laptop computer. Display and storage of the digital data is controlled using a custom-made graphical user interface based on LabVIEW software also incorporating digital filters and peak detection algorithms.

Within this user interface, real-time parameters are displayed such as the ECG trace, the respiration trace and the instant HR values extracted using a peak detection algorithm.

\section{B. Electrode interface}

A crucial consideration for the design of the smartmattress's front-end is the electrode material interface. Currently, there are several types of electrodes used for recording electrophysiological measurements. The most common interface that has been extensively used is based on silver/silver chloride $(\mathrm{Ag}-\mathrm{AgCl}) \quad[9,10]$. The main drawbacks of using $\mathrm{Ag}-\mathrm{AgCl}$ electrodes are that it requires skin preparation (i.e. removing skin oils) and the use of conductive gels as they require to make electrical contact with the sample.

In contrast, the EPS sensor only requires making physical contact with the patient without the need of any type of gel or skin preparation. Therefore, it is not affected by variations due to contact resistance in the same way that occurs within conventional electrodes.

Figure 2 shows the electrode-sensing interface of the EPS sensor deposited (see 'though' representation) on fabric. Screen printing techniques were used to provide complete freedom for the design layout as it does not need to follow the fabric structure. The procedure was carried out by screenprinting several layers of silver conductive paste and a UVcurable interface polymer paste onto the mattress's fabric. An interface layer was printed on the fabric before the silver ink was applied to improve the layer adhesion. The use of polymer interface layers have been proven to be able to effectively improve the durability of the electrode-sensing interface, in particular to withstand bending, stretching, abrasion and washing [13].

The interface paste UV-IF-1004 (smart Fabric Inks Ltd) creates a smooth surface on the fabric after solidification achieved by UV curing for a period of 30 s after printing. Cotton was chosen as the fabric to avoid triboelectric charging effects that could affect the accuracy of the sensor, The silver ink used in this work is a screen-printable paste Fabink-TC-C4001 (Smart Fabric Inks Ltd.) which was cured at $120 \mathrm{deg} \mathrm{C}$ for 10 minutes to solidify and evaporate the unwanted solvents. This provided good conductivity to maintain the SNR performance. The silver ink mainly consists of silver flakes, vinyl resin and a solvent. Silver flakes have been proven to be biocompatible and suitable for skin according to [14]

In addition to the electrode-sensing interfaces, conductive tracks were also printed on the fabric to connect the EPS sensors and the analogue processing units. A UV-curable waterproof paste (UV-IF-1039) was printed on the conductive tracks for encapsulation. This provides benefits on the reusability of the device as the mattress could be cleaned using the approved wipes used at the hospital for hygienic purposes without affecting the stability of the sensor front-end.
The combination of screen printing techniques and the EPS hardware circuitry provides a reliable ECG sensor with excellent biocompatibility and comfort for the end user.

\section{EXPERIMENTAL PROCEDURE AND RESULTS}

We evaluate the smart mattress design using two scenarios: a baby mannequin that was modified to produce an ECG signal output as shown in Figure 2, and a proof of principle demonstration with a single young infant aged 18 months.

Within the first scenario, we aimed to develop a more controlled environment where the signals were generated using a cardiac rhythm simulator as a way of validating our technology (data not shown). This allowed for fine-tuning of the smart-mattress in preparation for the work carried out with the young infant (data not shown).

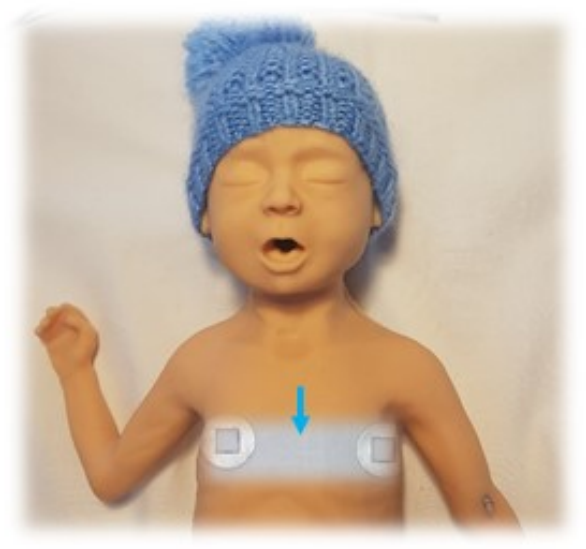

Fig. 2. Close-up view of the EPS sensor front-end showing a see through view of the silver deposited electrodes (blue arrow).

Within the second scenario, the experimental protocol was explained to the infant parents, who gave written informed consent before the study took place. All experiments were performed in accordance with relevant guidelines and regulations. The protocol was approved by the Science \&Technology Cross-Schools Research Ethics Committee (C-REC) University of Sussex with application ID number SOP/RGO/ER241/02.

The protocol was carried out in a private room and calm atmosphere to ensure recording stability. Recordings took place in one session, and the parents were asked to lay down the young infant on top of the sensor-integrated mattress. Ten ECG recordings lasting three minutes, where collected using our novel mattress design. Instant HRs, respiration rates and the ECG traces were recorded digitally for further analysis and representation. To validate the HR values obtained using our smart-mattress, parallel readings using a pulse oximeter (Nellcor, Minneapolis USA) were carried out.

As mentioned previously, PO measurements can take between 1 and 2 minutes to deliver a reliable reading once the sensor has been properly positioned [5], as well as not being suitable for preterm infants due to weak blood perfusion or whenever the HRs are below $100 \mathrm{bpm}$. In contrast with pulse oximetry, employing our proposed EPS device, we can reliably record additional functional parameters of the heart through the ECG trace and extract 
relevant data useful for diagnosing how cardiac chambers are behaving.

Figure 3 shows raw data of a single ECG pulse extracted non-invasively from the 18 months infant during the recordings. As it is shown, the ECG trace including the PQRS and T segments can be clearly observed without the requirement of further post processing. These waveform characteristics are useful for outlining the different stages of the heart functional activity such as atrial contraction ( $\mathrm{P}$ wave), ventricles contraction (QRS waves) and relaxation of the ventricles $(\mathrm{T})$.

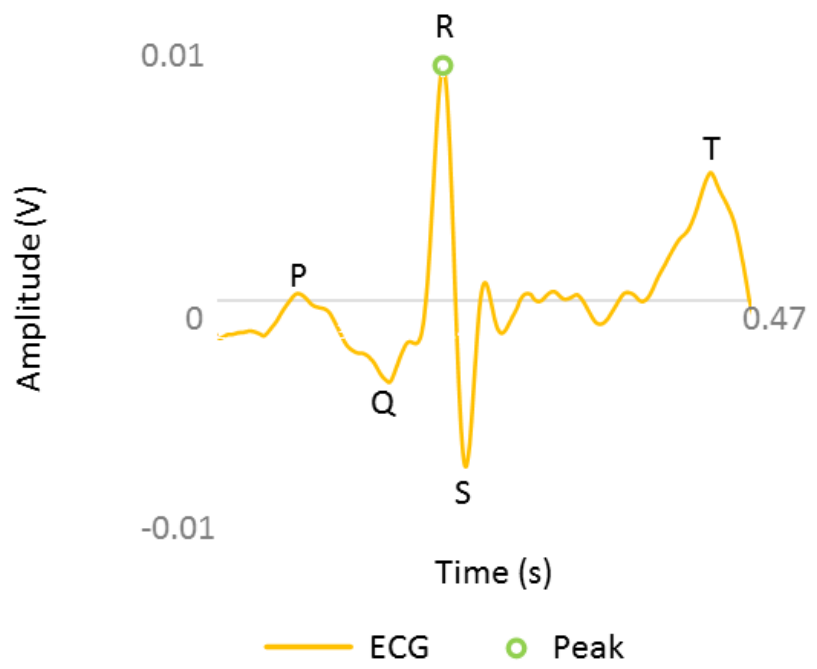

Fig. 3. Raw data of a single pulse extracted from the young infant during the tests showing detailed ECG characteristics such as the PQRS and $\mathrm{T}$ waves.

Figure 4 shows ten seconds of an ECG recording carried out with the infant together with the respiration traces.

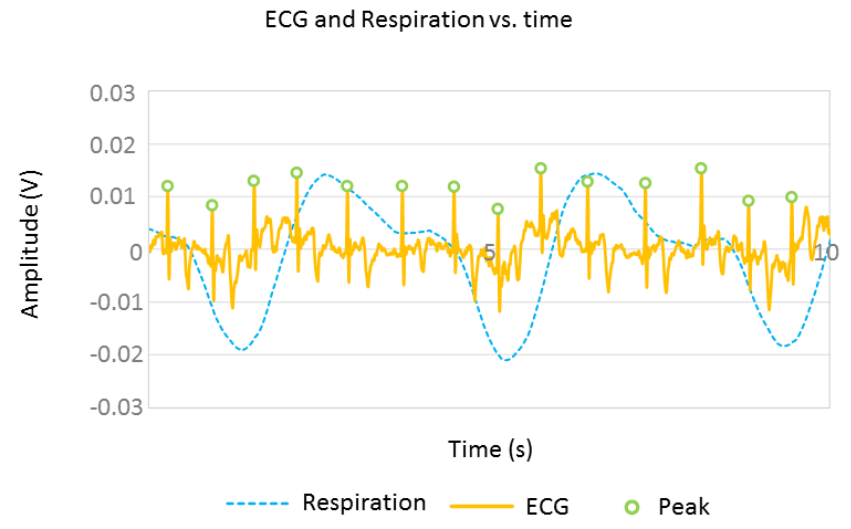

Fig. 4. Example ECG and respiration trace of ten seconds

Results show that the mean heart rate of the infant was $93.12 \pm 0.77$ this being within the normal heart rates at rest according to [15] and the mean respiration rate was $16.3 \pm$ 0.26 being in the lower limit of what has been reported in [16]. The parallel recordings using the pulse oximeter had an average value of $92.20 \pm 0.71$ falling within the range of measurements recorded using our sensing device. The data obtained is in agreement with the studies reported on $[15,16]$ for young infants HR and breathing rates at rest.
Our device provides accurate HR values in less than 30 seconds, once the baby is placed on top of the mattress. This is especially required for resuscitation purposes. Our time to get an ECG trace is up to four times faster than pulse oximetry, which is crucial to avoid long term effects related with poor oxygenation of organs within the baby.

Employing our smart- sensing mattress the detection of baby respiration rate is also possible. This is a crucial parameter required by the midwife carrying out the ventilation procedure. The heart rate together with the breathing rate will enable clinical staff to assess the success of the procedure in order to make quick and informed decisions.

\section{CONCLUSIONS}

In summary, we have presented a new sensing platform development based on the EPS technology to monitor the ECG activity of babies. The proof of concept demonstration conducted with a young infant confirms the potential of our smart-sensing mattress to be used during resuscitation procedures for term and preterm infants directly after birth.

Highly sensitive detection of ECG was accomplished using the proposed bespoke EPS sensor, even in the presence noise sources such as the mains of $50 \mathrm{~Hz}$ and without heavy post-processing or signal averaging techniques, thus preserving its high SNR. Both HR and the respiration rates were acquired in real time in less than 30 seconds, this being up to 4 times faster than pulse oximetry. The proposed technology has the potential to be used for new-born stabilization at the delivery room as it reduces the time to obtain the vital signs required for assessing the success of resuscitation procedures.

Additional applications include remote monitoring of infants in low income countries implementing a telemedicine system as described in [17]. Here, the midwife can evaluate the HR for assessing the ventilation procedure evolution, while doctors can remotely assess ECG to determine possible congenital disorders, if present.

Future directions of this research are focused on testing the device at the NICU with a cohort of ten infants once the device is certified to be used at the hospital and ethical approval regulations are completed.

\section{ACKNOWLEDGMENTS}

Authors would like to thank clinical staff for the valuable discussions carried out for the development of this work and access provided to the Brighton and Sussex Childrens Hospital. This work was funded by the University of Sussex Research Development Fund round 8.

\section{REFERENCES}

[1] O. D. Saugstad, S. Ramji, T. Rootwelt et al., "Response to resuscitation of the newborn: early prognostic variables," Acta Paediatr, vol. 94, no. 7, pp. 890-5, Jul, 2005.

[2] World Health organization 2017.

[3] C. O'Donnell, C. Kamlin, P. Davis et al., "Feasibility of and delay in obtaining pulse oximetry during neonatal resuscitation," Journal of Pediatrics, vol. 147, no. 5, pp. 698-699, NOV 2005, 2005.

[4] H. Mizumoto, S. Tomotaki, H. Shibata et al., "Electrocardiogram shows reliable heart rates much earlier than pulse oximetry during 
neonatal resuscitation," Pediatrics International, vol. 54, no. 2, pp. 205-207, APR 2012, 2012.

[5] D. Finn, G. B. Boylan, C. A. Ryan et al., "Enhanced Monitoring of the Preterm Infant during Stabilization in the Delivery Room," Front Pediatr, vol. 4, pp. 30, 2016.

[6] Kevat, D. Bullen, P. Davis et al., "A systematic review of novel technology for monitoring infant and newborn heart rate," Acta Paediatrica, vol. 106, no. 5, pp. 710-720, MAY 2017, 2017

[7] R. Prance and C. Harland, EPS Patent US8264247B2 (2012).

[8] P. Watson, R. J. Prance, S. T. Beardsmore-Rust et al., "Imaging electrostatic fingerprints with implications for a forensic timeline," Forensic Sci Int, vol. 209, no. 1-3, pp. e41-5, Jun, 2011.

[9] E. Rendon-Morales, R. J. Prance, and R. Aviles-Espinosa, "Noninvasive recordings of fetal electrocardiogram during pregnancy using electric potential sensors," AIP Advances Vol.8, pp. 105114, Oct, 2018 doi: 10.1063/1.5042358.

[10] E. Rendon-Morales, R. Prance, H. Prance et al., "Non-invasive electrocardiogram detection of in vivo zebrafish embryos using electric potential sensors," Applied Physics Letters, vol. 107, no. 19, NOV $92015,2015$.

[11] W. Gebrial, R. Prance, T. Clark et al., "Noninvasive imaging of signals in digital circuits," Review of Scientific Instruments, vol. 73, no. 3, pp. 1293-1298, MAR 2002, 2002.
[12] W. Gebrial, R. Prance, C. Harland et al., "Noninvasive imaging using an array of electric potential sensors," Review of Scientific Instruments, vol. 77, no. 6, JUN 2006, 2006.

[13] K. Yang, R. Torah, Y. Wei et al., "Waterproof and durable screen printed silver conductive tracks on textiles," Textile Research Journal, vol. 83, no. 19, pp. 2023-2031, NOV 2013, 2013.

[14] A.A. Chlaihawi, B.B. Narakathu, S. Emamian. B.J. Bazuin, M.Z. Atashbar. "Development of printed and flexible dry ECG electrodes," Sensing and Bio-Sensing Research, vol. 20, pp 9-15, SEP 2018, 2018.

[15] R. Kliegman et al., "Nelson Textbook of Pediatrics", 18th edition Elsevier, 2007.

[16] M. K. Marks, M. South, J. B. Carlin, "Reference ranges for respiratory rate measured by thermistry (12-84 months)," Archives of Disease in Childhood, vol 69, pp 569-572, 1993.

[17] L. Fanucci, S. Saponara, T. Bacchillone, M. Donati, P. Barba, I. Sanchez-Tato, C. Carmona. "Sensing Devices and Sensor Signal Processing for Remote Monitoring of Vital Signs in CHF Patients," IEEE Transactions on Instrumentation and Measurement, Vol. 62, no. 3, pp. 553-569, MAR 2013, 2013. 